\title{
Digital image correlation for microstructural analysis of deformation pattern in additively manufactured 316L thin walls ${ }^{1}$
}

\author{
Yanis Balit, Eric Charkaluk, Andrei Constantinescu \\ Laboratoire de mécanique des solides, CNRS, Ecole Polytechnique \\ Institut Polytechnique de Paris, \\ 91128 Palaiseau, France
}

\begin{abstract}
In additive manufacturing, the process parameters have a direct impact on the microstructure of the material and consequently on the mechanical properties of the manufactured parts. The purpose of this paper is to explore this relation by characterizing the local microstructural response via in situ tensile test under a scanning electron microscope (SEM) combined with high resolution digital image correlation (HR-DIC) and electron backscatter diffraction (EBSD) maps. The specimens under scrutiny were extracted from bidirectionally-printed single-track thickness 316L stainless steel walls built by directed energy deposition. The morphologic and crystallographic textures of the grains were characterized by statistical analysis and associated with the particular heat flow pattern of the process. Grains were sorted according to their size into large columnar grains located within the printed layer and small equiaxed grains located at the interfaces between successive layers. In situ tensile experiments were performed with a loading direction either perpendicular or along the printing direction and exhibit different mechanisms of deformation. A statistical analysis of the average deformation per grain indicates that for a tensile loading along the building direction, small grains deform less than the large ones. In addition, HR-DIC combined with EBSD maps showed strain localization situated at the interface between layers in the absence of small grains either individual or in clusters. For tensile loads along the printing direction, the strain localization was present
\end{abstract}

${ }^{1}$ Additive Manufacturing 31 (2020) 100862 https://doi.org/10.1016/j.addma.2019.100862 
in several particular large grains. These observations permit to justify the differences in yield and ultimate strength noticed during macroscopic tensile tests for both configurations. Moreover, they indicate that an optimization of the process parameters could trigger the control of microstructure and consequently the macroscopic mechanical behavior.

Keywords: Direct Energy Deposition, Digital Image Correlation, EBSD, Tensile Test, Microstructure

\section{Introduction}

Additive manufacturing (AM) has been defined in [1] as: "the process of joining materials to make objects from three-dimensional model data, usually layer upon layer". In the past decade, it has evolved from a rapid prototyping technique [2] to a manufacturing process for fully functional parts with a wide range of polymers and metallic materials [3]. Due to the low production rates, AM is more suitable for a small number of highly complex parts and therefore, applications in biomedical [4] and aerospace [5] industries are the most developed.

Two AM technologies have received particular attention in the past years, Powder Bed Fusion (PBF) and Directed Energy Deposition (DED). The PBF technology creates objects by sweeping a heat source over a given shape at the surface of a powder bed. As a layer of particles is fused, the powder bed descends and a fresh unfused layer of particles is distributed over the surface of the powder bed and the operations are then repeated. The DED technology is an additive manufacturing process in which the material is transported into a focused heat source and then deposited on the already constructed layers [6]. Particular variants of the DED process have been denoted in several ways in the literature such as: Direct Metal Deposition (DMD), 3D laser cladding, Laser Metal Deposition (LMD) and Direct Laser Deposition (DLD) to name a few. It has been used for various applications such as the manufacturing of complex or graded parts [7], for the deposition of coatings [8] or as a repairing technique [9]

The well known AISI 316L stainless steel is a suitable material for AM due to its welding capacity, its relatively high mechanical properties and for its high-temperature performance. In the last decade meaningful efforts were deployed by various groups to investigate the AM process for this steel and its inherent special mechanical properties. 
For example, the impact of the size and morphology of the powder was studied in [10] and it was found that over a certain particle diameter, the mechanical properties decrease. The influence of the process parameters, i.e. laser scanning speed and preheating temperature on the geometry and microstructure of a single-track metal deposition, were investigated in [11]. The conclusion was that the contact angle and the track height were controlled by the preheating temperature while the track width and the contact zone characteristics were governed by the scanning speed.

In [12], the authors showed that the best precision of large-scale parts produced by DED was obtained combining a small laser spot with high scanning velocity. Last but not least, the improvement of the surface finish of stainless steel thin walls was explored in [13], and it was noticed that by adjusting the scanning speed to a certain value of temperature in the melt pool, the layer's thickness reduces resulting in a better surface finishing.

The quality of the printed part depends on the process parameters. For DED, the powder flow, the speed deposition, the laser power and the lasing strategy have been defined as the main parameters steering the growth of the microstructure and therefore controlling the mechanical properties as reported in $[14 ; 15 ; 16]$.

The microstructure of the $316 \mathrm{~L}$ stainless steel and their influence on the mechanical performance were studied by various research groups at different material scales and through different investigation methods.

In [17], it was noted that the laser energy density has a strong influence over the grain size and the part density, hence affecting the mechanical properties. The manufacturing processes, the obtained microstructure and the mechanical and wear behavior were correlated in [18]. The results showed that the highest mechanical properties, understood as yield and ultimate tensile strength, as well as the best tribological performance were obtained for 316L stainless steel specimens produced by Selective Laser Melting (SLM) when compared to hot pressing or conventional casting. The result has been explained by a finer microstructure obtained in the AM process. In [19] is studied the effects of inter-layer dwell time interval and found that longer dwell time intervals imposed higher cooling rates, leading to finer microstructures and therefore to higher tensile strength and lower ductility.

Other efforts $[20 ; 21]$ tried to elucidate the direct relation between microstructure and observed mechanical properties, but due to the complexity of the phenomena and the large number of parameters involved, additional insights are needed to solve these questions. 
Indeed, it is well known that polycrystalline materials reveal microstructural strain heterogeneities due to the particular grain size, texture and morphology. These heterogeneities lead to strain localization, accumulation of local plastic strain $[22 ; 23]$ and are the precursors of crack initiation and propagation $[24 ; 25]$. Furthermore, in additive manufacturing, the lasing and building strategy will encourage the formation of oriented microstructures with elongated columnar grains [26]. In addition, anisotropic macroscopic properties are reported for example in [27] and [20].

In order to understand the anisotropic material behavior during tensile loading, it is necessary to unveil the particular deformation of the microstructure of AM materials and the underlying strain localization.

Advanced characterization techniques have emerged in the last decades, enabling the tracking of the deformation fields at the grain scale with applied loading by simultaneously combining digital image correlation (DIC) and Electron Backscatter Diffraction (EBSD) analysis [28]. Few investigations were performed at the scale of the grains for AM materials. For Ti-6Al$4 \mathrm{~V}$ manufactured by DED, the authors of [29] observed areas of high strain localisation at the interfaces of the layers. This accumulation was associated to the material anisotropy. The authors in [30] investigated the anisotropy of two INC718 specimens tested along and orthogonal to the building direction under tensile load. The associated strain maps at the grain scale of the specimens produced by SLM exhibited different patterns of deformation.

The goal of this paper is to investigate the strain heterogeneity and the strain localisation in the microstructure of $316 \mathrm{~L}$ specimens manufactured by DED during a tensile test. The observations are performed in situ under a scanning electron microscope (SEM) and combined simultaneously DIC and EBSD observations.

The paper is organized as follows. It starts with the presentation of the specimens and the experimental techniques. Afterwards, the characterization of the microstructure is discussed and the macroscopic mechanical properties are exhibited. Next, a procedure to compute the statistics of deformation of the microstructure at the grain scale is presented and the results are analyzed and discussed. Finally, several remarks will conclude the article. 


\section{Materials and Experiments}

A series of walls with single-track thickness have been manufactured by direct energy deposition (DED) of 316L stainless steel.

The commercially available powder was produced through gas atomization by Höganäs AB. The powder particles had a size within 45-90 $\mu \mathrm{m}$ and have their chemical and physical properties presented in the tables 1 and 2 respectively. The data were extracted from the certificate of powder analysis provided by the manufacturer and additional details can be retrieved from their website [31].

The 3D printer is a DED Mobile machine from BeAM [32] equipped with a 500W YLR-fiber laser. The powder is transported by argon gas flux and delivered to the deposition region through coaxial nozzles positioned approximately $3.5 \mathrm{~mm}$ above the substrate surface. The powder flow was directly measured by averaging the weight of the powder sent to the nozzle during 1 minute.

The single-track thickness walls were built employing a back-and-forth laser scanning strategy in the printing direction. In the building direction, the vertical spacing between the successive deposited layers was of $0.2 \mathrm{~mm}$. A schematic representation is proposed in Figure 1(a). Finally, the walls with the dimensions of $100 \mathrm{~mm} \times 30 \mathrm{~mm} \times 0.8 \mathrm{~mm}$ were printed on a $150 \mathrm{~mm} \times$ $5 \mathrm{~mm} \times 200 \mathrm{~mm} 316 \mathrm{~L}$ stainless steel substrate plate as displayed in Figure 2.

The calibration of the main writing parameters of the machine: laser power, deposition speed and powder flow were optimised using a parametric study having geometric details such as track and dilution height of the walls or global shape as final objective. The optimization process followed the procedure proposed in $[33 ; 14]$. More precisely, the appropriate set of parameters was chosen to ensure (i) a small dilution in the substrate to guarantee a metallurgical bond through the epitaxial growth between layers, (ii) a limited heat affected zone, (iii) a track to dilution height ratio between $1 / 5$ and $1 / 3$ and finally (iv) a large clad angle $\alpha$. This angle is a warrant of a low intertrack porosity in the case of multi-track volumes when overlapping is present. Therefore, it will not have any influence for the single-track thickness application studied next. The optimal set of parameters was the following: (i) a laser power of $225 \mathrm{~W}$, (ii) a powder flow of $6.5 \mathrm{~g} / \mathrm{min}$ and (iii) a deposition speed of $2000 \mathrm{~mm} / \mathrm{min}$.

The cross section observed by scanning electron microscope (SEM) of a single track on a $316 \mathrm{~L}$ sheet printed with the previous parameters and its 
complementary view obtained by Electron Backscatter Diffraction (ESBD) and are shown in Figure 3(a) and (b) respectively. One can remark an epitaxial growth of elongated grains from the interface and oriented towards the center of the deposited track. Moreover one can also observe the presence of small grains located close to the surface in contact with the air.

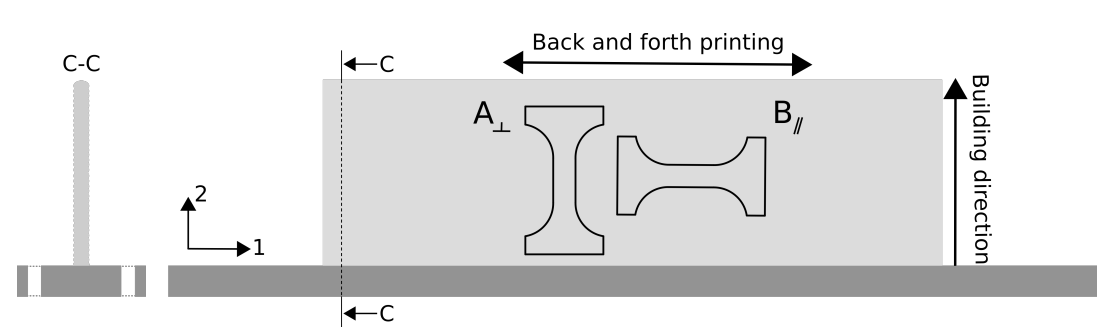

(a)

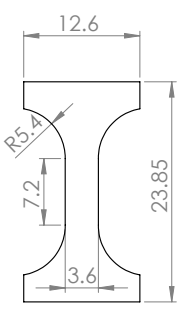

(b)

Figure 1: (a): Side view (C-C) of the single-track thickness wall printed on the substrate and position of the specimens $A_{\perp}$ and $B / /$, orthogonal and along the printing direction respectively. (b): Dimensions in minimeter $(\mathrm{mm})$ of the dogbone shaped specimens extracted from the wall.

Dogbone shaped specimens used for observation and testing were extracted from the single-track walls by water jet cutting with a Mach $2 b$ waterjet [34].

Their precise positions and geometry in the wall is displayed in Figure 1(a) and (b). The distorsion of the wall is negligible, i.e. the differences with a straight ruler are of the order of the magnitude of the roughness. Furthermore, the specimens are polished on the both side removing rugosity and any potential distorsion. Finally, the residual stress of the polished specimens were measured by X-ray diffraction and it was found that low compressive residual stress was present $75 \mathrm{MPa} \pm 31$. Let us further remark that larger residual stresses and stronger distortion have only been observed when higher walls have been build. The dogbone geometry does not adhere to any standards and was imposed by pratical limitations such as the size of the ion polisher chamber or compatibility requirements with the homemade SEM in situ tensile tests machine. However, a prior finite element study confirmed a homogeneous macroscopic strain in the zone of interest. The dimensions of the specimens were defined by the compatibility with the homemade SEM in situ tensile tests machine. Two orientations were defined for the specimens in order to apply tensile loading along the building and printing direction i.e, perpendicular and parallel with respect to the printing direction. Next, 


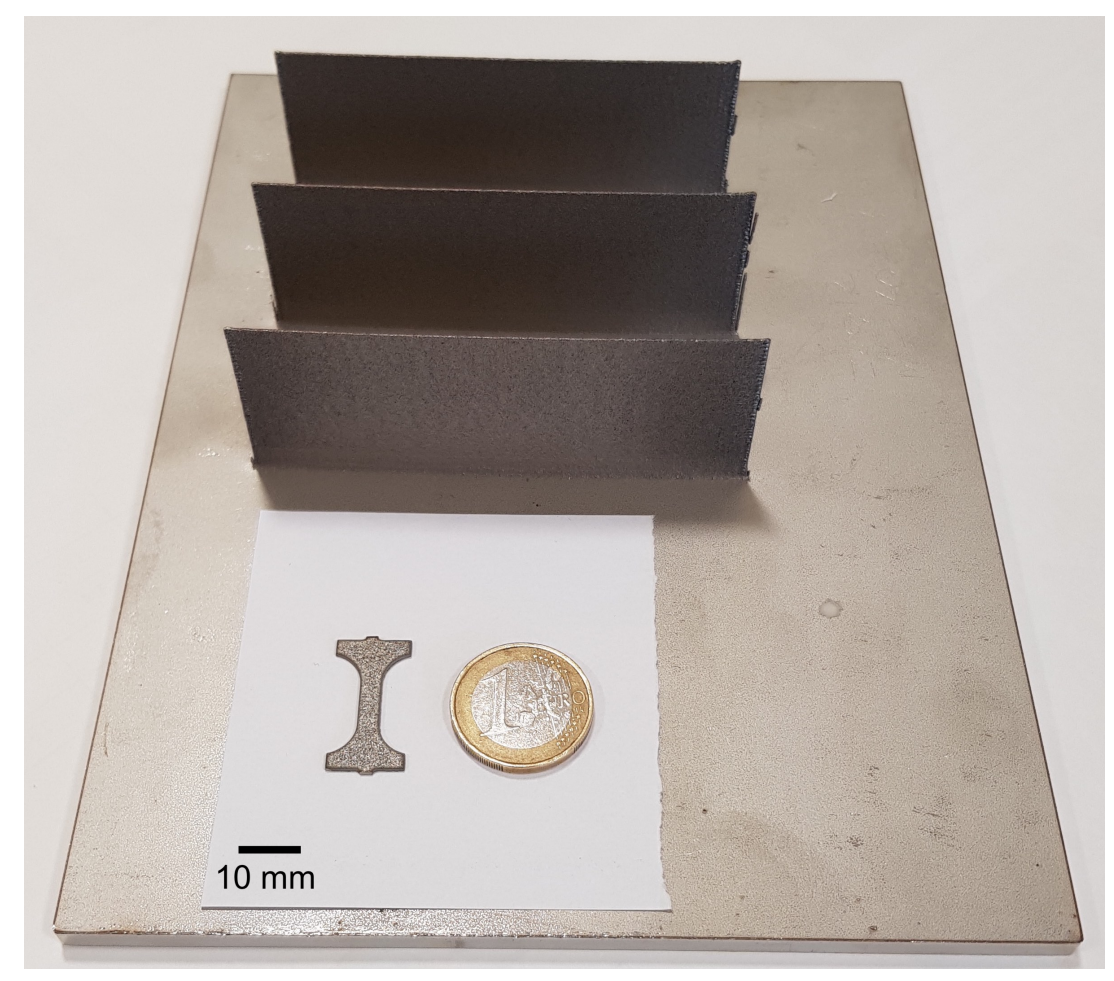

Figure 2: The printed wall on the substrate and a specimen after waterjet cutting.

we denote the specimen with a tensile direction perpendicular and parallel to the printing direction as $A_{\perp}$ and $B_{/ /}$respectively, as represented in Figure $1(\mathrm{a})$.

\begin{tabular}{|c|c|c|c|c|c|c|c|}
\hline Elements & $\mathrm{C}$ & $\mathrm{Mo}$ & $\mathrm{Ni}$ & $\mathrm{Fe}$ & $\mathrm{Mn}$ & $\mathrm{Cr}$ & $\mathrm{Si}$ \\
\hline Weight percent & 0.011 & 2.5 & 12.7 & balance & 1.5 & 16.9 & 0.7 \\
\hline
\end{tabular}

Table 1: Chemical properties of the $316 \mathrm{~L}$ powder.

The specimens were mechanically polished after the extraction in order to obtain a mirror surface adequate for SEM observations. As such, the specimens lost their surface roughness inherited from the manufacturing process. However, this will only affect final failure or fatigue properties but not the ones discussed next. The polishing was performed with silicon carbide abrasive papers with successive grit values from 400 to 4000 and finished with a $1 \mu \mathrm{m}$ diamond paste. The specimens were finally ion polished during one 


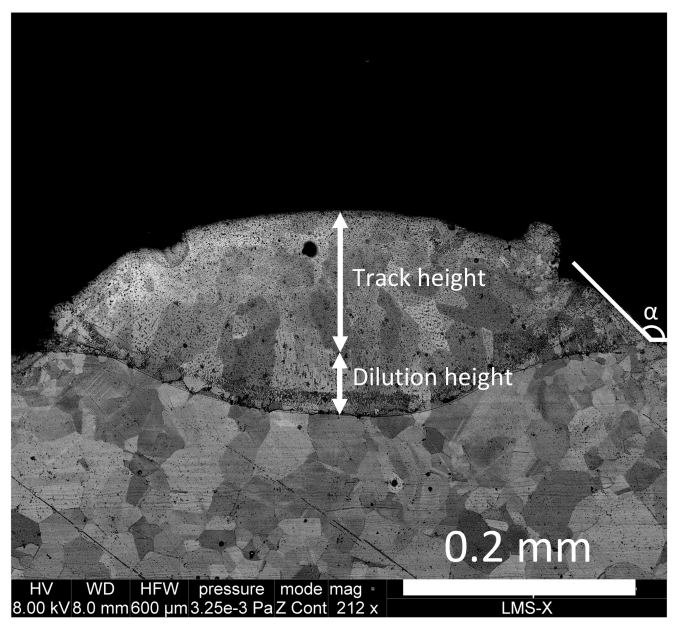

(a)

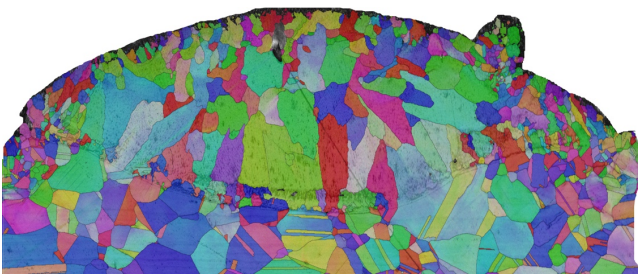

\section{$0.2 \mathrm{~mm}$}

Figure 3: (a): SEM image of the track with its geometry parameters. (b): ESBD of the track showing epitaxial growth of long grain from the substrate and small grain on the superior contour.

\begin{tabular}{|c|c|}
\hline Apparent density (Hall) & Flowrate (Hall) \\
\hline $3.96 \mathrm{~g} / \mathrm{cm} 3$ & $18.1 \mathrm{sec} / 50 \mathrm{~g}$ \\
\hline
\end{tabular}

Table 2: Physical properties of the $316 \mathrm{~L}$ powder.

hour with the following parameters: $6^{\circ}$ polishing angle, $6 \mathrm{keV}$ electron beam power, $6 \mathrm{rpm}$ specimen angular velocity in a PECS II machine from Gatan [35].

The microstructure of the specimens was examined by EBSD (Electron Backscatter Diffraction) on a FEI QUANTA 600F SEM (Scanning Electron Microscopy) apparatus. Data was collected and analyzed using the Aztec and Channel 5 software respectively. EBSD maps were collected on a central area of $1.8 \times 1.9 \mathrm{~mm}^{2}$ for specimen $A_{\perp}$ and $1.8 \times 1.85 \mathrm{~mm}^{2}$ for the specimen $B_{/ /}$using a step size of $1 \mu \mathrm{m}$ in both cases.

In order to record displacement and strain fields at different scales by Digital Image Correlation (DIC), a multi-scale lithography grid was performed on each specimen in a $3 \times 3 \mathrm{~mm}^{2}$ central area. The lithography preparation consists of applying a drop of PMMA $5 \%$ (i.e. Poly(methyl methacrylate) on the polished specimen surface and to spread it by spin coating (2000 rpm) during 70 seconds. The specimens with the transparent thermoplastic 
coating are then put into an oven at $170^{\circ} \mathrm{C}$ during 30 minutes (polymerizing conditions). Subsequently, the electron beam of the SEM is driven on the surface specimen by the software Raith Elphy Quantum to reproduce the PMMA designed pattern by irradiating the resin. After this step, the specimens are bathed during 90 seconds in a solution (1/4 methyl-ethal-cetone and $3 / 4$ propanol) to dissolve the irradiated resin. Then, gold is firstly deposited by sputtering $(1 \mathrm{~nm})$ and by vacuum vaporization $(12 \mathrm{~nm})$. Finally, the non-irradiated resin is removed by ultrasonic cleaning during 1 minute with ethyl-acetate.

The specimens were tested in SEM under a tensile load in order to observe the strain field at the microstructure scale and to associate it with EBSD maps. The strain rate was fixed at $2 \times 10^{-4} \mathrm{~s}^{-1}$ and the loading steps $1,2,3,4$ and 5 were respectively defined by the macroscopic strain levels corresponding to $1 \%, 6 \%, 12 \%, 16 \%$ and $20 \%$. The loading steps were imposed based on a real-time strain computation based on DIC on the larger grid, with a cross spacing of $100 \mu m$ (blue grid on Figure 4). At each strain step, an elastic unloading is performed and an image of the smaller grid area, with a $3 \mu \mathrm{m}$ cross spacing (red grid on Figure 4) is recorded. The comparison of this image with the initial one provided a high resolution strain full-fields and permits to analyse the deformation process at the grain scale, highlighting the zones with a high plastic strain.

In order to compare the obtained mechanical properties with other experiments, an additional monotonic tensile test, i.e. in the absence of the unloading steps, was equally performed on the same tensile machine. The in situ machine was associated with a $4 K N$ load cell and the strain rate was fixed at $10^{-3} s^{-1}$.

Digital image correlation (DIC) for strain fields computation based on successive images of deformed specimens is now a conventional technique for the measurement of strain fields as discussed in [36] and [37]. However as different algorithms and assumptions are covered by software and literature, we shall summarize the procedure used next. Displacement full-fields are computed using Correl Manu, a homemade software [22; 38; 39].

The correlation starts from a set of non-overlapping points located on the grid crosses of the reference image, $C D_{n}\left(X_{n}, Y_{n}\right)$, on Figure 5(a). This set of points, also denoted as correlation domains, is then tracked in its new position $\left(C D_{n}\left(y_{n}, y_{n}\right)\right)$ through the images at different deformation steps, see the deformed configuration on Figure 5(b). The tracking is done by 


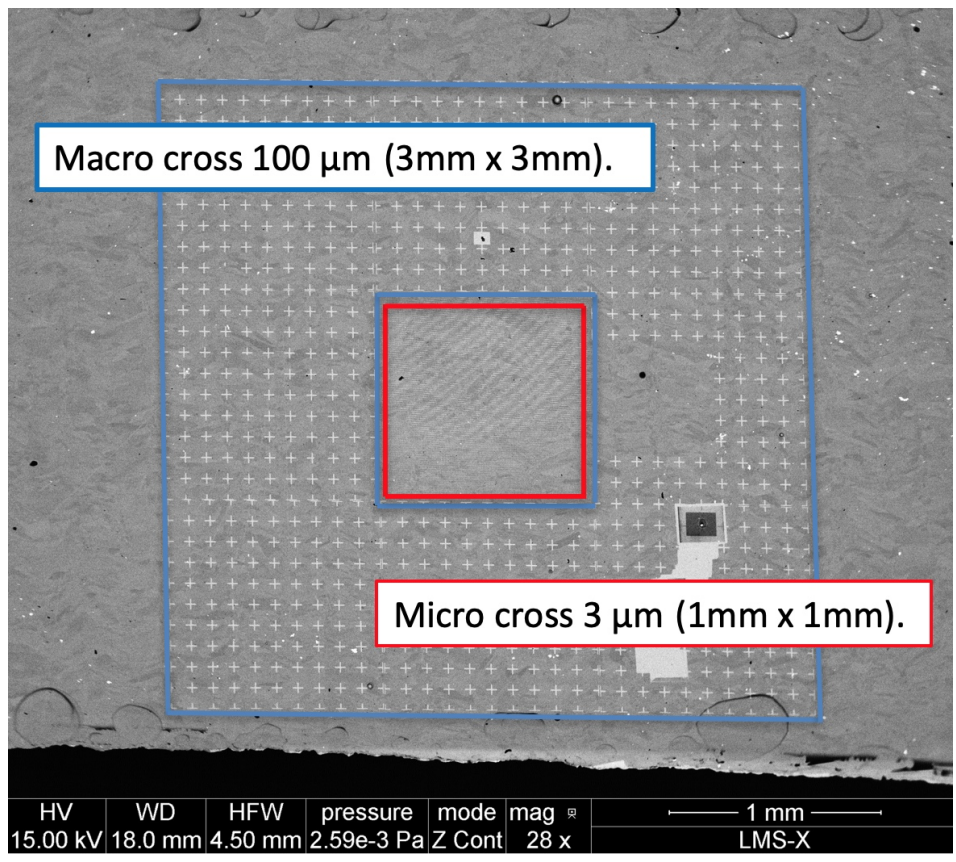

Figure 4: Image of the multiscale grids on the tensile specimen. Blue area of $3 \times 3 \mathrm{~mm}^{2}$ with macro crosses with a pitch of $100 \mu m$ was designed for macroscopic deformation patterns while the red area of $1 \times 1 \mathrm{~mm}^{2}$ was designed to capture high resolution images needed for the DIC and thus to determine the deformation pattern at the microstructure scale

minimization of the correlation coefficient, which measures closeness of the distribution of grey levels between reference and deformed images.

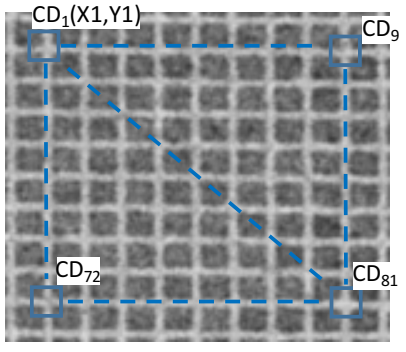

(a)

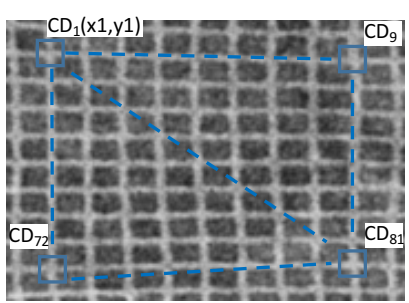

(b)

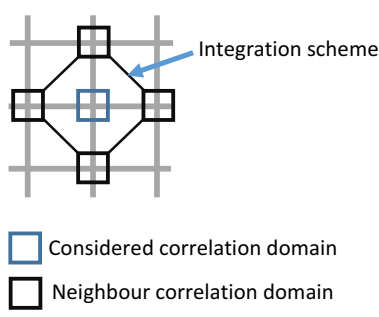

(c)

Figure 5: Layout of the correlation domain (a) in the reference image, (b) in the deformed image and (c): scheme of the integration domains for computation of the the GreenLagrange strain tensor. 
The local in-plain Green-Lagrange $\mathbf{E}_{\Omega}$ tensor for each correlation point is calculated over an integration domain $\Omega$, defined by the neighboring correlation points as displayed in Figure 5 (c). The average displacement gradient $\langle\mathbf{F}\rangle_{\Omega}$ over the domain $\Omega$, is computed as detailed in [38].

The representation of the average displacement gradient $\langle\mathbf{F}\rangle_{\Omega}$ in Cartesian components is:

$$
\langle\mathbf{F}\rangle_{\Omega}=F_{i j} \mathbf{e}_{i} \otimes \mathbf{e}_{j}
$$

where $\mathbf{e}_{i}$ are the unit vectors of the Cartesian coordinates. Its components oriented along the $X$ and $Y$ axes are expressed as:

$$
\begin{gathered}
F_{X X}=\frac{1}{2 S} \sum_{n=1}^{N}\left(x^{n}+x^{n+1}\right)\left(Y^{n+1}-Y^{n}\right) \\
F_{X Y}=\frac{1}{2 S} \sum_{n=1}^{N}-\left(x^{n}+x^{n+1}\right)\left(X^{n+1}-X^{n}\right) \\
F_{Y X}=\frac{1}{2 S} \sum_{n=1}^{N}\left(y^{n}+y^{n+1}\right)\left(Y^{n+1}-Y^{n}\right) \\
F_{Y Y}=\frac{1}{2 S} \sum_{n=1}^{N}-\left(y^{n}+y^{n+1}\right)\left(X^{n+1}-X^{n}\right)
\end{gathered}
$$

where $S$ denotes the area of the integration domain and $\mathrm{N}$ the number of correlation domains. $\left(x^{n}, y^{n}\right)$ and $\left(X^{n}, Y^{n}\right)$ are the coordinates of the control points in the actual and initial configuration respectively.

From the displacement gradient, the Green-Lagrange strain tensor is computed using the convential formula:

$$
\mathbf{E}_{\Omega}=\frac{1}{2}\left(\langle\mathbf{F}\rangle_{\Omega}^{T} \cdot\langle\mathbf{F}\rangle_{\Omega}-\mathbf{I}\right)
$$

Next, the experiments will be performed under a small strain assumption and therefore:

$$
\varepsilon \approx \mathbf{E}_{\Omega}
$$

The correlation errors of this method, see [38], can be reduced significantly by increasing the size of the integration domain (at the expense of local informations). In this work, as we are interested in the mean deformation 
of a grain, this implies that the larger the grain is, the smaller the error will be. More precisely, the estimated maximum absolute error for the $\varepsilon_{11}$ strain component is $26 \times 10^{-4}$ for a $8 \times 8 \mu \mathrm{m}^{2}$ area corresponding to an average small grain and $36 \times 10^{-5}$ for a $32 \times 32 \mu m^{2}$ area which is equivalent to an average large grain. The terms small and large grain will be explained further. Moreover, the errors are also related to the noise of the recorded images by the SEM and can be reduced by decreasing the acquisition speed [40]. In the cases studied here, we had to balance the dwell time with the image resolution, because a longer exposition time would enhance the image quality but will distort the acquisition. To ensure a reasonable grey contrast for the DIC, it was necessary to fix the resolution to $8192 \times 7168$ pixels i.e. the smallest resolution still allowing a few pixels within the micro crosses of the red area in case of a Horizontal Field Width $(\mathrm{HFW})=1750 \mu \mathrm{m}$.

\section{Results and discussion}

\subsection{Grain morphology and texture analysis}

The microstructure was analyzed qualitatively and quantitatively in terms of grain size, shape and distribution from the EBSD data.

The statistical analysis of grain sizes and shapes conducted to similar results for both specimens irrespective of their orientations as displayed in table 3 . This result was expected as a consequence of the following factors: (i) both specimens were extracted in the middle section of the wall, i.e. away from the extreme free edges and at the same height of the wall, thus avoiding the eventual variation of spatial and temporal characteristic of the temperature, (ii) they have the same level of polishing, i.e same thickness and (iii) the areas where EBSD analysis are performed are large enough to contain a statistically representative number of grains. As a consequence, in order to simplify the discussion, only the microstructural analysis conducted on the specimen $B_{/ /}$will be reported here in the sequel.

The microstructural texture, in terms of both morphology and crystallographic orientations is determined by the local heat flow during solidification and cooling. The history will guide the preferred growth directions of the grains. The particular heat flow pattern depends on both the geometry and heat exchange coefficients as well as of a given set of printing parameters such as the power of the laser, the printing speed, the scanning pattern strategy etc. which are driving the position of the heat source in the transient heat 
flow problem. Direct relations between the printing parameters and the microstructural texture are discussed in [41; 42;43], but will not be presented in detail in this paper.

A typical microstructure at the center of the wall is displayed in Figure 6(a). For the given vertical build step of $0.2 \mathrm{~mm}$ one layer of grains has an average height equal $0.12 \mathrm{~mm}$ and its grains have an average orientation of $61^{\circ}$ with respect to the printing direction (more precisely $\alpha_{1}=58.6^{\circ}$ and $\alpha_{2}=63.6^{\circ}$ as shown on Figure $6(\mathrm{~b})$. The noticeable zigzag pattern is a consequence of the alternating movement of the laser head during the manufacturing, which controls the local thermal history and the direction of maximum heat flow in different layers [41].

The crystallographic texture obtained from the EBSD analysis was plotted in Figure 7 using three pole figures corresponding to different areas of focus. Two subsets of grains corresponding to two successive layers were extracted, one relative to the layers printed from left-to-right and the other one relative to layers printed from right-to-left. Both are represented in blue and red respectively in Figure 7(a). In Figure 7(b), the pole figure of the complete set of grains is plotted while, in Figure 7(c) and (d) is displayed the two subsets of grains. In Figure 7(c), the crystallographic texture of the layers printed from left-to right is represented and one can remark a hotspot close to the Building Direction (BD) axe which illustrates a slight texture of the crystallographic plane $\{100\}$. A similar observation can be made in Figure 7 (d) for the layers printed from right-to-left. By firstly analyzing the subsets, it is easier to understand the whole EBSD texture as the sum of two fiber textures. The distance of the two hotspots from the $\mathrm{BD}$ axe corresponds to a tilt angle of $30^{\circ}$ toward the printing direction i.e. the crystallographic and morphologic are both aligned. It is known that the texture is determined during solidification and in the case of centered cubic alloys [41], it is defined by the preferred crystallographic direction which is closely aligned with the maximum heat flow. The precise direction of the heat flow was not measured during printing, but the observed textures gave a clear idea of the heat flow pattern in this case.

A precise observation of the Figure 6(a) exhibits an important surface covered by large grains. They mainly form the printed layers while smaller grains will cover the interface between the layers. The smaller grains will equally cluster between layers as illustrated in Figure 6(b). However a repetitive pattern of the small grain clusters could not be found. For example, clusters are present in interfaces I5 and I4, while absent in I1, I2 and I3. 


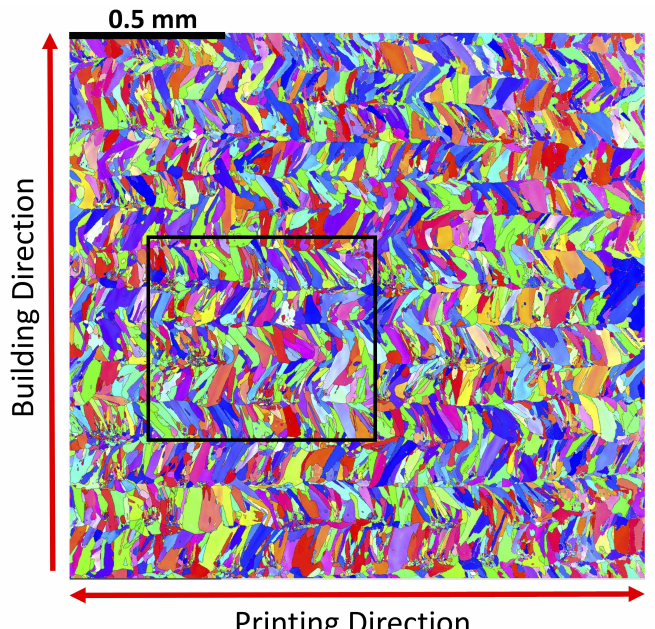

Printing Direction

(a)

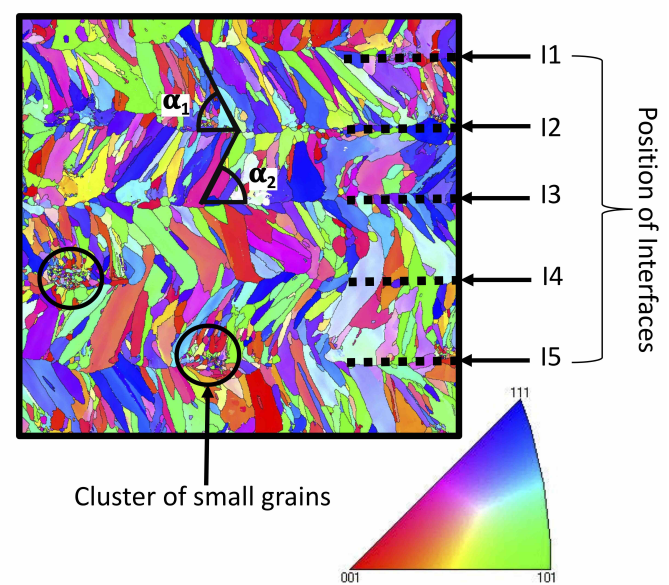

(b)

Figure 6: (a): EBSD of the specimen $B_{/ /} \cdot(\mathrm{b})$ : Zoom on the EBSD showing in details the interfaces between layers, cluster of small grains present at interfaces and the morphological grain angles for both direction of printing.

Similar observations on the presence of small grains and their clustering between layers was also reported in [43]. More precisely, the authors related this type of microstructure to particular values of the solidification rate and the thermal gradient associated with the heat suction from the substrate and the printing parameters. They reported that after a specific building height, the fine grains tend to disappear because of the heat accumulation in the built part, which corresponds to our observations. It has also been previously reported in [27], that grains at the top of the wall are larger than the ones at the bottom. This was associated to a faster cooling closer to the substrate. However, this was not observed in the present work as the studied surface was too small and positionned at the center of the wall.

Next, we propose to sort the grains in two families with respect to their surface area, i.e. small and large grains. This threshold value between the two families correspond to the surface of the biggest grain found in a cluster of small grains and is equal to an area of $176 \mu \mathrm{m}^{2}$. This area approximately corresponds to a circle with a $15 \mu \mathrm{m}$ diameter.

This separation will allow to better apprehend the role of different grains in the deformation process and asses from a statistical point view the differences in term of size, quantity and surface occupied by grains, as resumed 


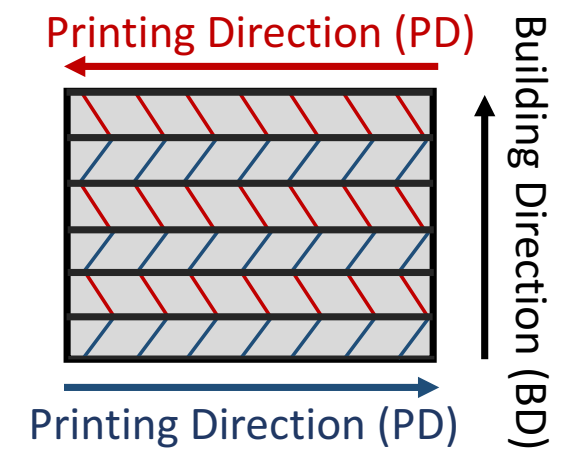

(a)

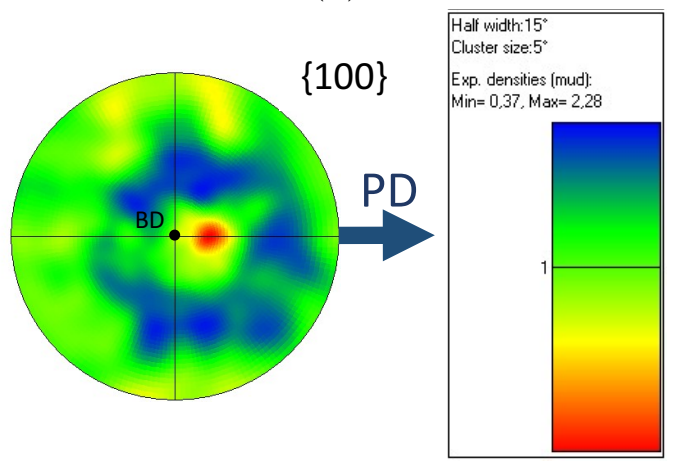

(c)

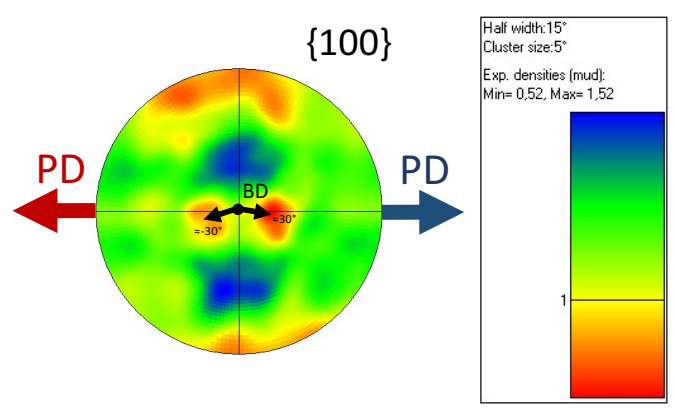

(b)

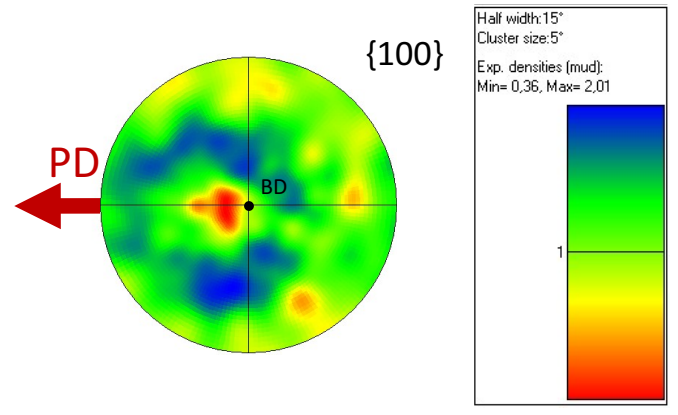

(d)

Figure 7: The texture of the microstructure: (a) Schematic representation of the printed layers and their relative morphological grains angles associated by color to the printing direction. Pole Figure for the whole EBSD, for the layers printed from left to right the one printed from right to left diplayed in (b), (c) and (d) respectively

in the table 3 . One can easily note that there are more small grains, $61 \%$ of total number, than large one, $39 \%$, while the small ones occupies only $8 \%$ of the total surface. We have a ratio of 4 between the average diameters of large and small grains.

The aspect ratio of the shape, was defined as the ratio of principal axes of the smallest circumscribed ellipse, implying that aspect ratio of 1 indicates an equiaxed grain. Ellipses fitting the small grains have an average aspect ratio of $2.4 \pm 1.4$ while ellipses fitting the large grains have a an average aspect ratio of $3.4 \pm 2.1$ (complete data set is available in Table 3). The distribution of the grain sizes is depicted in Figure 8 by the plot of the continuous probability density function (PDF) of frequency as a function 


\begin{tabular}{|c|c|c||c|c|}
\hline & \multicolumn{2}{|c||}{ Specimen A } & \multicolumn{2}{c|}{ Specimen B } \\
\hline & small grains & large grains & small grains & large grains \\
\hline Average diameter & $7.6 \pm 3.3 \mu \mathrm{m}$ & $31.6 \pm 16.8 \mu \mathrm{m}$ & $7.2 \pm 3.4 \mu \mathrm{m}$ & $29.6 \pm 15.4 \mu \mathrm{m}$ \\
\hline Mediane of diameter & $7 \mu \mathrm{m}$ & $26.3 \mu \mathrm{m}$ & $6.5 \mu \mathrm{m}$ & $24.4 \mu \mathrm{m}$ \\
\hline Numbers of grains & $61 \%$ & $39 \%$ & $67 \%$ & $33 \%$ \\
\hline Surface occupied & $8 \%$ & $92 \%$ & $11 \%$ & $89 \%$ \\
\hline Average of the aspect ratio & $2.4 \pm 1.4$ & $3.4 \pm 2.1$ & $2.6 \pm 1.6$ & $3.8 \pm 3.2$ \\
\hline Mediane of the aspect ratio & 2 & 2.8 & 2.1 & 3.2 \\
\hline EBSD surface & \multicolumn{2}{|c|}{$3.3 \mathrm{~mm}^{2}$} & \multicolumn{2}{c|}{$3.4 \mathrm{~mm}^{2}$} \\
\hline
\end{tabular}

Table 3: Grains size statistics.

of aspect ratio for small and large grains. The continuous distribution was fitted from the discrete measured distribution using [44]. Moreover, one can see that, the PDF of the aspect ratio of small grains is narrow attaining its peak value at an aspect ratio of 1.8 which is smaller than 2.4, its average aspect ratio. The distribution of aspect ratios for the large grains is more spread, it attains its peak at an aspect ratio of 2.2 which is also smaller than 3.4 , the average aspect ratio for large grains. Moreover, we recall that the total surface of small grains is far smaller than the surface occupied by large grains while the small grains are superior in terms of quantity.

As a closure remark regarding sizes and shapes, we can state that large grains have a columnar shape while small grains have a more equiaxial one.

\subsection{Mechanical properties}

The tensile engineering stress versus strain curve of the specimens $A_{\perp}$ or $B_{/ /}$are compared in Figure 9. In order to provide a precise quantitative comparison of the tensile response differences, we provide standard material parameters such as the yield stress at $0.2 \%$ (YS), ultimate strength (UTS) and the ductility (D). To facilitate the analysis, the ratio $R$ of the material parameters of $B_{/ /}$over $A_{\perp}$ is compared to values from literature $[45 ; 20 ; 46]$. If the ductility is almost similar for the specimen $A_{\perp}$ or $B_{/ /}$with a ratio of 1 , the value of the ultimate strength of specimen $B_{/ /}$is higher with a ratio attaining 1.18 and a similar trend is observed for the yield strength with a ratio of 1.4. One can therefore notice an anisotropy for the yield and the ultimate strength of the two specimens.

Previous publications $[20 ; 47 ; 48]$ on stainless steel employing DED as a manufacturing technology present similar trends regarding the mechanical behavior under uniaxial tensile test. The tensile engineering stress versus strain curve of the present specimens $A_{\perp}$ or $B_{/ /}$are compared with the curves 


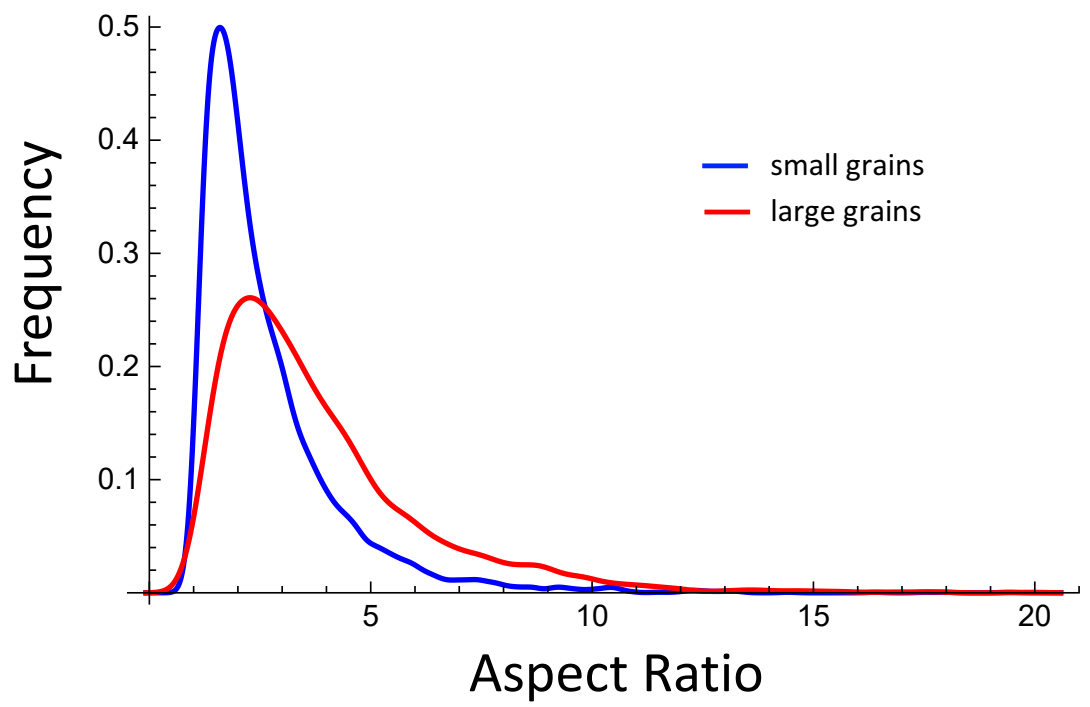

Figure 8: Probability density function of grain ellipticity, i.e. frequency in terms of ratio between the large and small axes of the ellipse fitting the grain by its surface and shape.

of their homologous one extracted from a multi-track thickness volume in 316L obtained by DED [45]. One can notice that the differences between the tensile properties for both set of configuration are comparable, in the sense that one obtains higher yield and ultimate strength for the type $A_{\perp}$ specimens and longer ductility for the type $B_{/ /}$specimens. Nevertheless, it can be noted that intrinsic values are different. Even tough the nature of the feedstock (316L stainless steel) and printing technology (DED) are the same, the size and chemical composition of the powder as well as the machines parameters (laser power, speed deposition, printing strategy etc.) for both compared studies are different. In [49], the authors summarized the tensile properties of 316L specimens manufactured by DED from different research groups permitting to highlight the scatter of the properties and the large spectrum of tensile strength. In addition, it was also noted that specimens manufactured with lower linear heat inputs have smaller melt pools, thus faster cooling rate leading to a finer microstructure and therefore higher yield and ultimate tensile strengths in comparaison to the ones produced with higher linear inputs. Also, it was reported that even for a same linear heat inputs, the type of laser has an influence on the energy absorbed by the substrate or deposited layers and thus on the final properties. In addition, 
the chemical composition [50] and particle size [51] of the powder affect the mechanical properties as well as the printing parameters as reminded in the introduction. Therefore, one can perceive the sensitivity of the mechanical properties to process/machine's parameters and can consequently understand the difference observed in Figure 9 and more generally between studies of the same type of materials additively manufactured.

Furthermore, the material parameters of table 4 are compared with the ones of a hot rolled and annealed 316L. The hot rolled and annealed material present isotropic properties with a higher ductility but lower yield and ultimate strength in comparison to 316L obtained by DED. An explanation for the anisotropy in the case of DED was proposed in [27] who analyzed a material manufactured in similar conditions as our specimen. A specimen of type $A_{\perp}$ has long and narrow columnar grains oriented along the building direction. Therefore, under uniaxial test along the building direction, dislocations are required to cross fewer grain boundaries to elongate the sample than specimen in the configuration of $B_{/ /}$where dislocations need to cross more boundaries. This can explain the difference between the UTS and YS. It was also noticed in [20] that interlayer in case of a tensile direction orthogonal to the printing direction are ideal crack initiation sites. In order to validate such assumptions, it is therefore important to investigate the strain localization process at the microstructure scale as proposed in the next section.

\begin{tabular}{|c|c|c|c|c|c|c|c|c|c|}
\hline Technology & \multicolumn{3}{|c|}{ YS (MPa) } & \multicolumn{3}{c|}{ UTS (MPa) } & \multicolumn{3}{c|}{ D (\%) } \\
\hline Printing versus tensile direction & $\perp$ & $/ /$ & $R$ & $\perp$ & $/ /$ & $R$ & $\perp$ & $/ /$ & $R$ \\
\hline DED (our results)-from wall & $272 \pm 8$ & $381 \pm 5$ & 1.4 & $621.7 \pm 9.4$ & $734.1 \pm 12.8$ & 1.18 & $46.4 \pm 2$ & $44.4 \pm 3.8$ & 0.96 \\
\hline LENS [45] from bulk & 479 & 576 & 1.2 & 703 & 776 & 1.1 & 46 & 33 & 0.72 \\
\hline LMDS[20] from wall & 352 & 558 & 1.6 & 536 & 639 & 1.19 & 46 & 21 & 0.46 \\
\hline Hot rolled and annealed [46] & 302 & 307 & 1.01 & 595 & 600 & 1 & 57 & 59 & 1.03 \\
\hline
\end{tabular}

Table 4: Mechanical properties of 316L from the present tests and from the literature $[45 ; 20 ; 46] . \quad R$ is the ratio of the material parameters of the specimen $B_{/ /}$over the specimen $A_{\perp}$

\subsection{Combined strain and EBSD analysis}

Next, a combined DIC-EBSD analysis is proposed to reveal and to understand the observed differences between the mechanical properties in tensile test, depending on the orientation of the loading with respect to the printing direction, i.e. along or orthogonal to printing direction. The analysis is split in several steps: (i) an initial EBSD analysis of the central part of the 


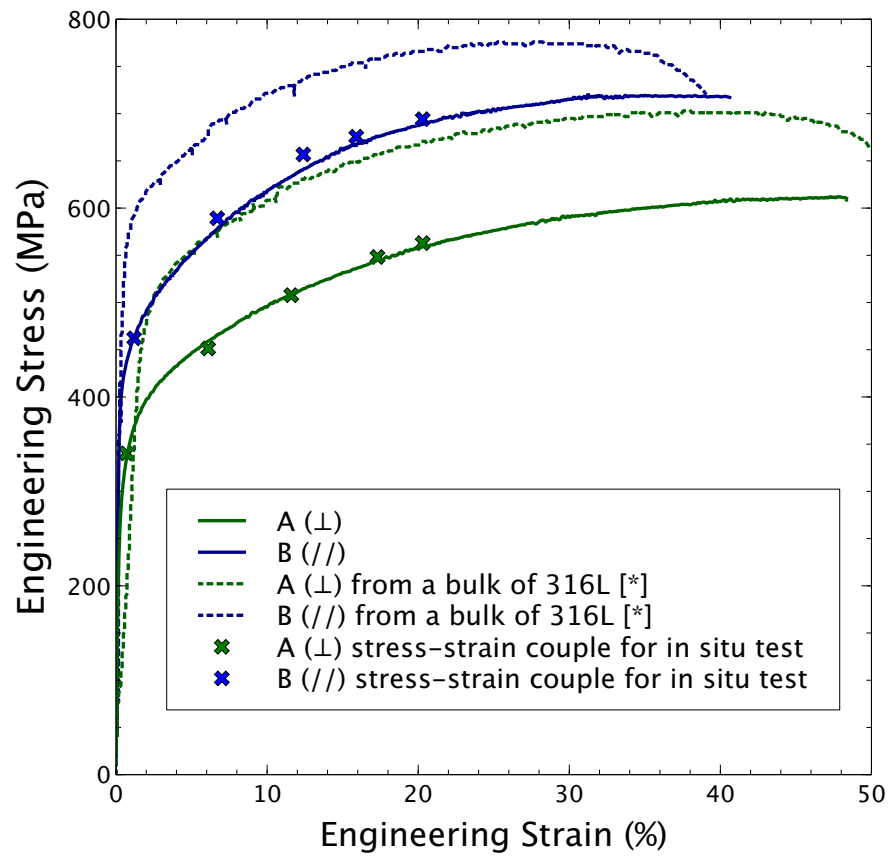

Figure 9: Monotonic tensile stress-strain curves of the specimen $A_{\perp}$ and $B_{/ /}$extracted from a DED 316L wall and their counterparts extracted from DED 316L bulk. * $=[45]$. The stress-strain position data for the in situ tests of specimen $A_{\perp}$ and $B_{/ /}$are represented by green and blue crosses respectively.

specimen as described in Figure 4, (ii) a SEM in situ tensile test combined with DIC described in section 2, (iii) a projection of DIC results on EBSD which will be described in this section and (iv) a statistical analysis of mean strain value per grain associated to a study of the local strain patterns.

As stated previsously, DIC full-fields were projected on EBSD maps: this enables us to determine the correlation points included in each grain. To perform this overlapping, we had to correct the non-linear distortion of the EBSD coming from several factors such as the beam drift, the stability of the specimen etc. The procedure is detailed in [52] and the code used for this correction was adapted from the Matlab procedure available in [53].This image correction procedure will distort the initial image shape from a rectangular to an arbitrary shape as already reported in [52]. It is important to notice that the distortion is image specific as it depends on the specific defor- 
mation patterns of microstructure, i.e. texture, grainsize,etc. and therefore the exact transformation will be different for each image. A similar behavior can be observed in the images exhibited in Figure 14. A Matlab routine was programmed to reconstruct the EBSD map with a unique RGB color averaged for each grain based on its euler angle in order to determine the contour of the grain and thus, the correlation points included. Then, as presented previously, from the average transformation gradient, it was possible to compute the mean value of the Green-Lagrange tensor components in each grain. The precise procedure is schematized in Figure 10.

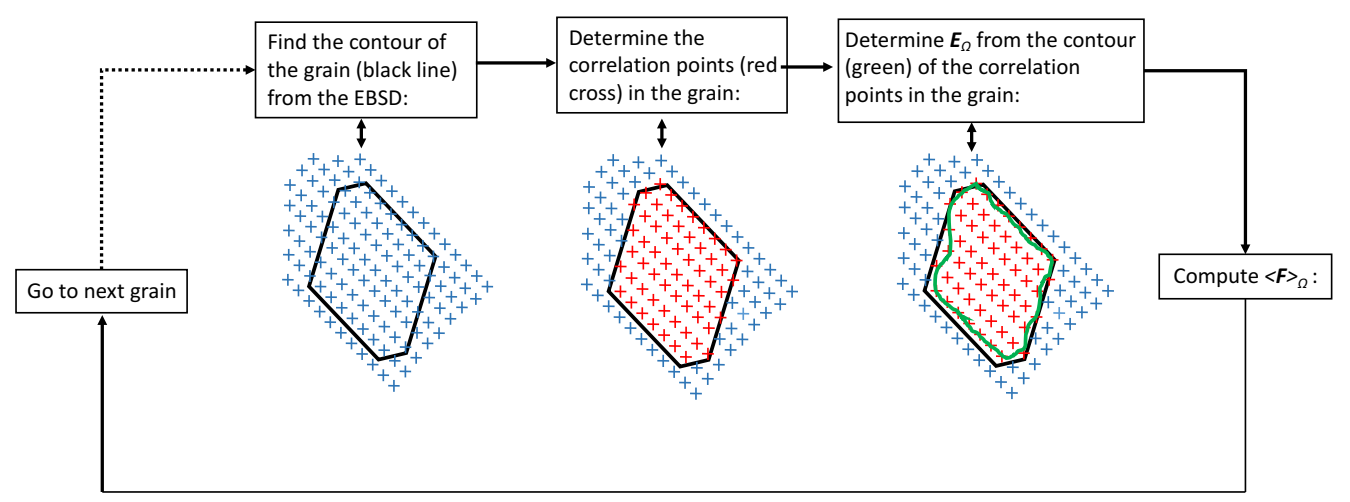

Figure 10: Algorithmic scheme of the implemented procedure for the determination the mean value of the Green-Lagrange tensor components in each grain.

First of all, a comparative study of the two grain families (small and large) for specimens $A_{\perp}$ and $B_{/ /}$is proposed. Let us mention that the deformation patterns/deformation statistics are defined starting with the first applied strain step and evolve afterwards mainly in terms of strain amplitude. Therefore, only the data for the step 2, corresponding to a global strain of $\approx 6 \%$ will be discussed.

\begin{tabular}{|c|c|c||c|c|}
\hline & \multicolumn{2}{|c||}{ Specimen $A_{\perp}$} & \multicolumn{2}{c|}{ Specimen $B_{/}$} \\
\hline & small grains & large grains & small grains & large grains \\
\hline Average of the strains & $0.052 \pm 0.025$ & $0.06 \pm 0.019$ & $0.072 \pm 0.031$ & $0.074 \pm 0.026$ \\
\hline Mediane of the strains & 0.048 & 0.06 & 0.069 & 0.072 \\
\hline
\end{tabular}

Table 5: Grains size statistics.

In Figure 11, the PDF of the $\varepsilon_{11}$ strain component relative to the grain's family (red for large grains and blue for small grains) is plotted in case of a 
global applied strain of $\approx 6 \%$ (purple line). For the specimen $A_{\perp}$, the two distributions are quite different with, for the large grains, a peak aligned with the global strain whereas for the small grains a peak of frequency centered around $4,8 \%$. In addition, the strain average of the small grain is $5,2 \%$ with a median at 4,8\% while for the large grains, the average and median were $6,9 \%$ and $7,2 \%$ respectively. Thus, one can note that when the tensile test is perpendicular to the printing direction, the mechanical behavior is not the same for small and large grains: small grains deform less than large grains.

The same investigation for specimen $B_{/ /}$led us to a different conclusion. One can remark on Figure 11(b) that both peaks are aligned on the global mean strain. More precisely, the average strain of the small grain is $7.2 \%$ with a median at $6.9 \%$ while for the large grains, the average and median were values $7.4 \%$ and $7.2 \%$ respectively. One can therefore conclude that when the tensile load is parallel to the printing direction, the mechanical behavior is almost the same for small and large grains.

In order to get a precise understanding of the relation between grain size and average grain deformation, we propose to analyze the probability density function of the grain surface versus average grain strain over the $\varepsilon_{11}$ strain component (2D PDF). This representation will permit to highlight where the maximum deformation is concentrated and will indicate where strain localization and/or failure will probably occur. The corresponding colored histograms are displayed in Figure 12. In Figure 12(a) and (c), one can see the $2 \mathrm{D} \mathrm{PDF}$ for the small and large grain of the specimen $A_{\perp}$ respectively at the same step of deformation as previously. From these plots, we cannot add additional details to the conclusion made in Figure 11(a). Nevertheless, for the specimen $B_{/ /}$, particular areas are observable in the 2D PDF of the large grains as shown in Figure 12(d). These areas are circled by white dots and they correspond to particular large grains that deform more than the others. This was hidden in the figure 11(b), where just from this plot, deformation could have been thought uniform. As a first step into the investigation to determine the difference observed during the tensile tests, one can infer that for the specimen $A_{\perp}$, there is a difference of the tensile behavior between small and large grains while for the specimen $B_{/ /}$, some particular large grains are highly localizing.

It is now necessary to investigate the spatial localization (i.e the deformation patterns) from the strain field obtained by the DIC overlapped on the EBSD in order to qualify and explain the previous statistical observations. In Figure $13(\mathrm{a})$ and (b), the local $\varepsilon_{11}$ strain component obtained by DIC for 
specimen $A_{\perp}$ and $B_{/ /}$is displayed in the case of a global strain of $6.1 \%$ and $6.7 \%$ respectively. One can remark in Figure 13(a) that the strain is very localized in some areas while on the Figure 13(b), the strain localisation is more spread in some columnar shape allowing to recognize the zig-zag pattern of the microstructure. For the step of deformation 2, the DIC full-fields for $A_{\perp}$ and $B_{/ /}$were respectively overlapped on their relative EBSD map in Figure 14(a) and (b) in order to associate the strain pattern with the microstructure. One can see in Figure 14(a) that the strain localisations actually correspond to interfaces between the layers. Nevertheless, it can also be noted that not all interfaces show such localization and one can observe that these areas correspond to zone where there is the presence of small grains and clusters of small grains. One can remember that for specimen $A_{\perp}$, the statistical analysis showed that small grains deform less. Therefore, it is reasonable that no strain localisation is observed at the interface in the presence of clusters of small grains. Finally, this confirms the results of [20] which noticed that interlayer is a crack initiation area, when the tensile direction is orthogonal to the printing direction.

A similar investigation of the DIC overlapped on the EBSD for the specimen $B / /$ is plotted in Figure 14(b); in this case, the strain localization follows a zig-zag pattern of particular large grains. In addition, there are no singularities at interfaces and this makes sense considering the previous results showing that small and large grains present the same strain distribution. The strong localization in some particular large grains shown in 14(b) was also expected since it was highlighted by the 2D PDF in Figure 11(d). A complementary graph is proposed in Figure 15 where the average $\epsilon_{11}$ per grains is plotted and confirmed the presence of particular large grains with higher localisation. Finally, the same trend of localisation for higher deformation steps is observable as displayed in Figure $14(\mathrm{c}),(\mathrm{d}),(\mathrm{e})$ and (f). It can also be noted that for $A_{\perp}$, localization seems to be more present at the boundaries of the grains while for $B_{/ /}$, it is more diffuse.

In conclusion, in the case of a tensile load orthogonal to the printing direction, the small grains deform less than the large ones. It was also observed that the strain localization is mainly situated at the interface between layers in the absence of small grains either individual or in clusters. In the other case of a tensile load along the printing direction, we found that small and large grains deform the same. We also observed that the strain localization was present in some particular large grains. 


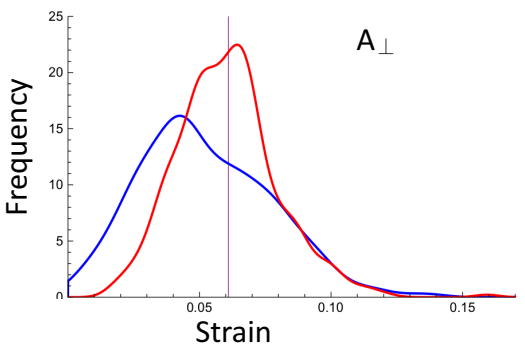

(a)

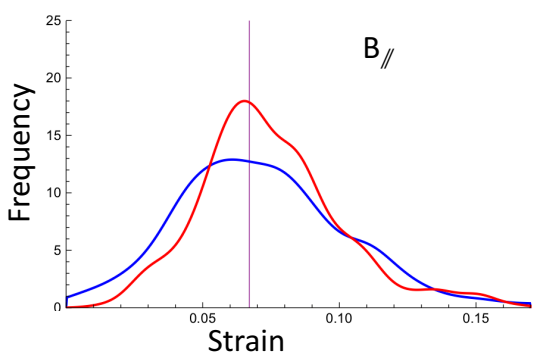

(b)

Figure 11: Probality density function of the strain associated for the small and large grains of specimen $A_{\perp}$ and $B_{/ /}$in (a) and (b) respectively. 


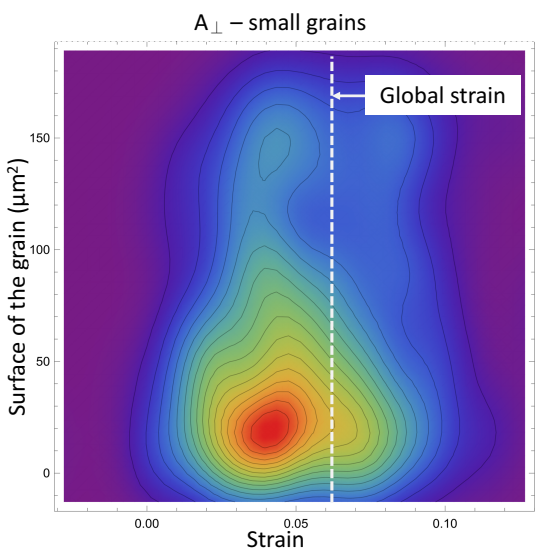

(a)

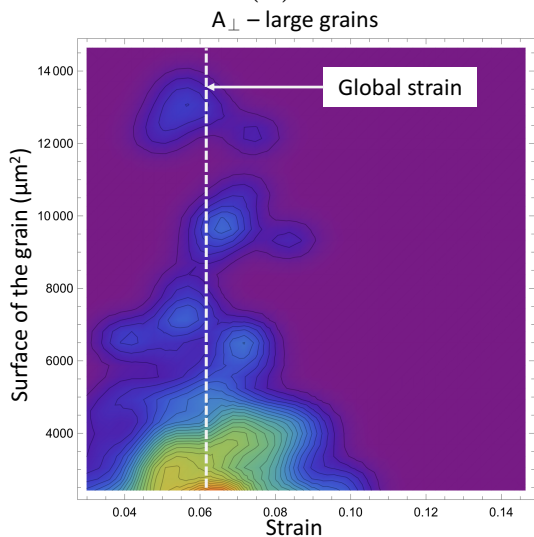

(c)

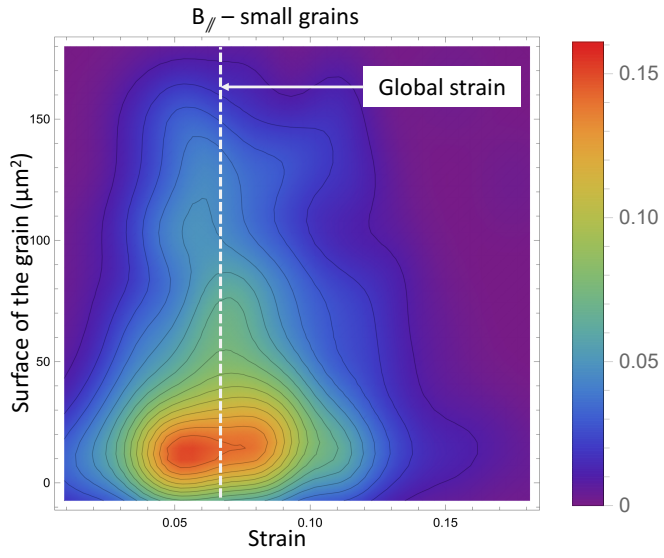

(b)

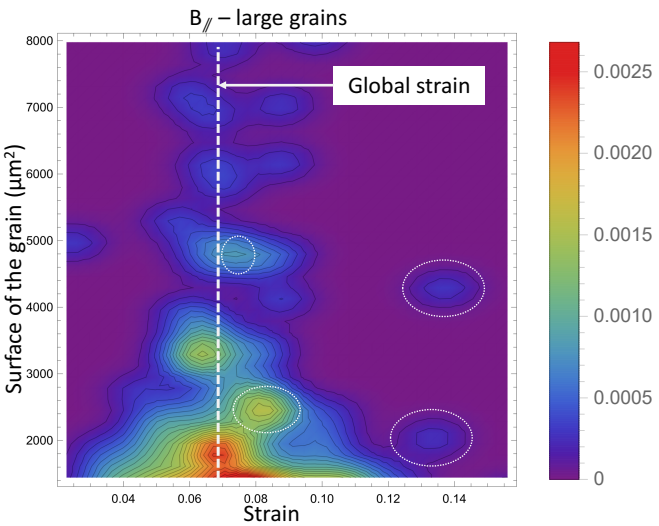

(d)

Figure 12: 2D Probality density function of the strain for the small of specimen $A_{\perp}$ and $B_{/ /}$in (a) and (b) respectively and for their large grains in (c) and (d). 


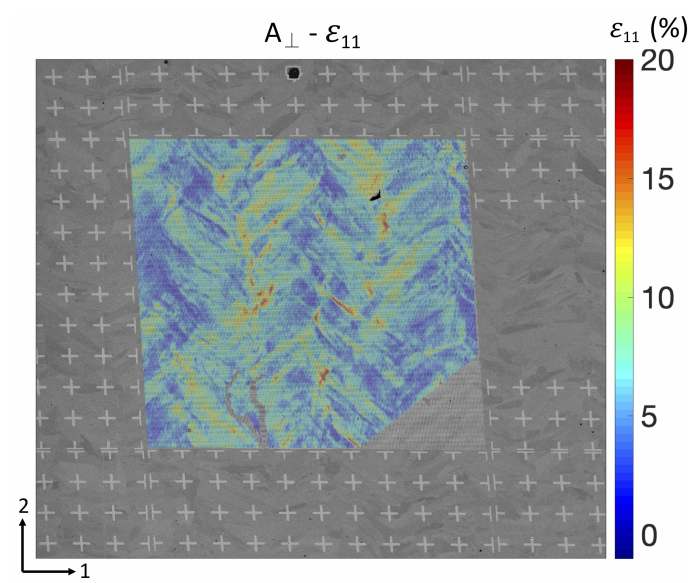

(a)

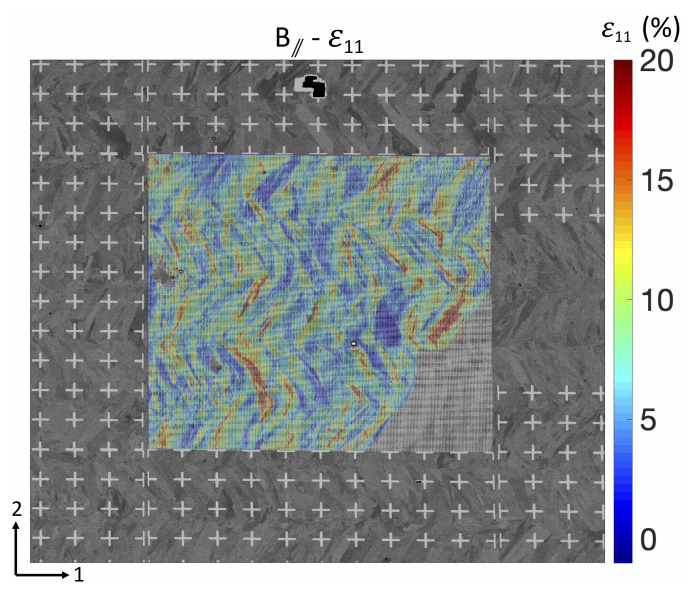

(b)

Figure 13: Evolution of the horizontal deformation $\varepsilon_{11}$ obtained by digitial image correlation for the specimen $A_{\perp}$ and $B_{/ /}$in (a) and (b) respectively. 


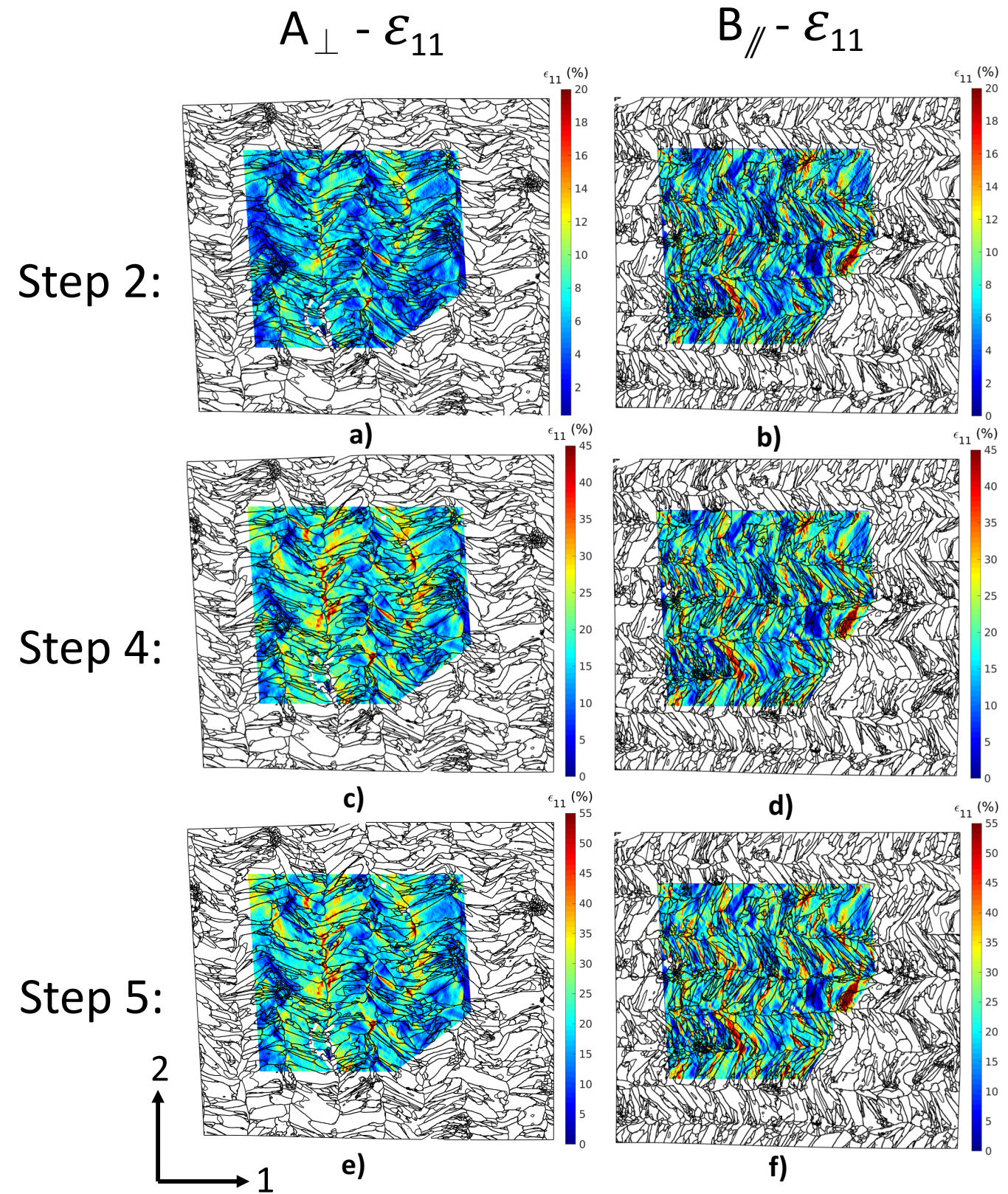

Figure 14: DIC overlapped for $A_{\perp}$ and $B_{/ /}$on their respective corrected EBSD maps at the step of deformation 2,4 and 5 corresponding to macroscopic strain of $6 \%, 16 \%$ and $20 \%$ respectively. 


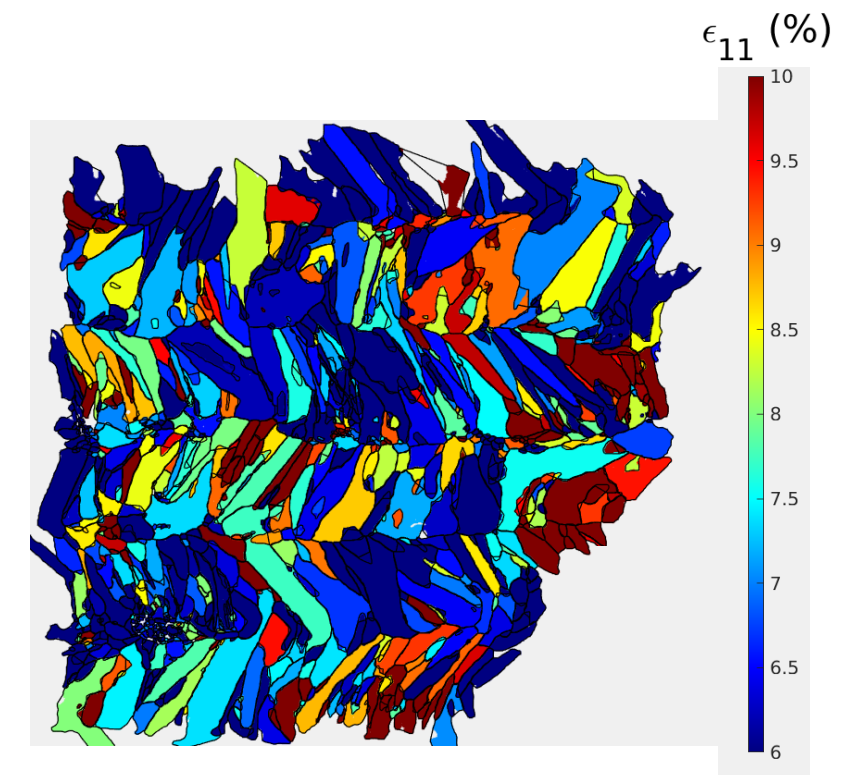

Figure 15: Average $\epsilon_{11}$ per grains for the specimen $B_{/ /}$at the deformation step 2. 


\section{Conclusion and Perspectives}

In this study, we associated the observed mechanical anisotropy of specimens produced by additive manufacturing with the anisotropic and heterogeneous microstructure and the induced microscopical strain localization. Specimens were extracted from 316L stainless steel single-track thickness walls built by DED. Firstly, we investigated microstructure through morphologic and texture analysis. Then, we performed tensile tests that revealed anisotropy. Finally, in situ tensile tests under an SEM combined with HR-DIC and EBSD maps enabled the qualification and quantification of the mechanisms of deformation. The conclusions are the following:

- The morphologic and crystallographic textures are aligned and can be associated with the heat flow pattern induced by the printing strategy.

- The grain morphology is characterized by small equiaxed grains present as isolated or in clusters at the interface between printed layers and large columnar grains within the layer.

- Anisotropy was detected during tensile tests. Specimen tested along the printing direction $\left(B_{/ /}\right)$exhibited higher mechanical properties in terms of yield strength (YS) and ultimate tensile strength (UTS) than the specimen tested perpendicularly to the printing direction $\left(A_{\perp}\right)$. $\mathrm{A}$ similar trend was reported in literature.

- In the case of a tensile load perpendicular to the printing direction, small grains deform less than large ones. Moreover, strain localization is mainly situated at the interface between layers in the absence of small grains.

- In the case of a tensile load along the printing direction, the strain localization was observed in some particular large grains.

There are several natural extensions of this work such as a precise investigation of the slip systems of the grains exhibiting strong deformations, and

the observations of the mechanisms of deformation under cyclic loadings and their relations with the fatigue phenomena. 


\section{Acknowledgement}

This work was financed by the DGA (France) and the SNCF (France). The authors would like to thank Louis Romain Joly (SNCF), Eric Lafontaine (DGA) and Marie-Christine Sainte Catherine (DGA) for fruitful discussions on the subjects, as well as Sylvain Durbecq, Pascal Marie, Alexandre Tanguy, Simon Hallais and Hakim Gharbi for accompanying the printing of the structures and the SEM measurements. 
[1] ASTM International. Standard terminology for additive manufacturing technologies. Technical report, ASTM International, 2012.

[2] R.M. Miranda, G. Lopes, L. Quintino, J.P. Rodrigues, and S. Williams. Rapid prototyping with high power fiber lasers. Materials and Design, 29(10):2072 - 2075, 2008.

[3] Nannan Guo and Ming C. Leu. Additive manufacturing: technology, applications and research needs. Frontiers of Mechanical Engineering, 8(3):215-243, Sep 2013.

[4] Sneha Prabha Narra, Peter N. Mittwede, Sandra DeVincent Wolf, and Kenneth L. Urish. Additive manufacturing in total joint arthroplasty. Orthopedic Clinics of North America, 50(1):13 - 20, 2019. New Technologies.

[5] R. Liu, Z. Wang, T. Sparks, F. Liou, and J. Newkirk. 13 - aerospace applications of laser additive manufacturing. In Milan Brandt, editor, Laser Additive Manufacturing, Woodhead Publishing Series in Electronic and Optical Materials, pages 351 - 371. Woodhead Publishing, 2017.

[6] International Organization for Standardization. Iso/astm 52900, additive manufacturing - general principles - terminology. Technical report, International Organization for Standardization, 2015.

[7] Taewoo Hwang, Young Yun Woo, Sang Wook Han, and Young Hoon Moon. Functionally graded properties in directed-energy-deposition titanium parts. Optics and Laser Technology, 105:80 - 88, 2018.

[8] Niyanth Sridharan, Ercan Cakmak, and Ryan R. Dehoff. Microstructure evolution during laser direct energy deposition of a novel fe-cr-ni-w-b hardfacing coating. Surface and Coatings Technology, 358:362 - 370, 2019.

[9] J. Michael Wilson, Cecil Piya, Yung C. Shin, Fu Zhao, and Karthik Ramani. Remanufacturing of turbine blades by laser direct deposition with its energy and environmental impact analysis. Journal of Cleaner Production, 80:170 - 178, 2014. 
[10] Andrew Pinkerton and K Mahmood. Direct laser deposition with different types of $316 \mathrm{l}$ steel particle: A comparative study of final part properties. Proceedings of the Institution of Mechanical Engineers Part B Journal of Engineering Manufacture, 227:520-531, 042013.

[11] I. Yadroitsev, P. Krakhmalev, I. Yadroitsava, S. Johansson, and I. Smurov. Energy input effect on morphology and microstructure of selective laser melting single track from metallic powder. Journal of Materials Processing Technology, 213(4):606 - 613, 2013.

[12] Mingming Ma, Zemin Wang, Dengzhi Wang, and Xiaoyan Zeng. Control of shape and performance for direct laser fabrication of precision largescale metal parts with 3161 stainless steel. Optics and Laser Technology, 45:209 - 216, 2013.

[13] Masoud Alimardani, Vahid Fallah, Mehrdad Iravani-Tabrizipour, and Amir Khajepour. Surface finish in laser solid freeform fabrication of an aisi 3031 stainless steel thin wall. Journal of Materials Processing Technology, 212(1):113 - 119, 2012.

[14] V. Ocelík, U. de Oliveira, M. de Boer, and J. Th M de Hosson. Thick Cobased coating on cast iron by side laser cladding: Analysis of processing conditions and coating properties. Surface and Coatings Technology, 201(12):5875-5883, 2007.

[15] H.Y. Wan, Z.J. Zhou, C.P. Li, G.F. Chen, and G.P. Zhang. Effect of scanning strategy on mechanical properties of selective laser melted inconel 718. Materials Science and Engineering: A, 753:42 - 48, 2019.

[16] Hussam El Cheikh, Bruno Courant, Samuel Branchu, Xiaowei Huang, Jean Yves Hascot, and Ronald Guilln. Direct Laser Fabrication process with coaxial powder projection of 316L steel. Geometrical characteristics and microstructure characterization of wall structures. Optics and Lasers in Engineering, 50(12):1779-1784, 2012.

[17] Di Wang, Changhui Song, Yongqiang Yang, and Yuchao Bai. Investigation of crystal growth mechanism during selective laser melting and mechanical property characterization of 3161 stainless steel parts. Materials \& Design, 100:291 - 299, 2016. 
[18] F. Bartolomeu, M. Buciumeanu, E. Pinto, N. Alves, O. Carvalho, F.S. Silva, and G. Miranda. 3161 stainless steel mechanical and tribological behavior - a comparison between selective laser melting, hot pressing and conventional casting. Additive Manufacturing, 16:81 - 89, 2017.

[19] Aref Yadollahi, Nima Shamsaei, Scott M Thompson, and Denver W Seely. Effects of process time interval and heat treatment on the mechanical and microstructural properties of direct laser deposited 3161 stainless steel. Materials Science and Engineering: A, 644:171 - 183, 2015 .

[20] Kai Zhang, Shijie Wang, Weijun Liu, and Xiaofeng Shang. Characterization of stainless steel parts by laser metal deposition shaping. Materials and Design, 55:104 - 119, 2014.

[21] Xianglong Wang, Jose Alberto Muñiz-Lerma, Oscar Sánchez-Mata, Mohammad Attarian Shandiz, and Mathieu Brochu. Microstructure and mechanical properties of stainless steel 3161 vertical struts manufactured by laser powder bed fusion process. Materials Science and Engineering: A, 736:27 - 40, 2018.

[22] E. Héripré, M. Dexet, J. Crépin, L. Gélébart, A. Roos, M. Bornert, and D. Caldemaison. Coupling between experimental measurements and polycrystal finite element calculations for micromechanical study of metallic materials. International Journal of Plasticity, 23(9):1512 1539, 2007.

[23] M Sachtleber, Z Zhao, and D Raabe. Experimental investigation of plastic grain interaction. Materials Science and Engineering: A, 336(1):81 $-87,2002$.

[24] Michael D. Sangid. The physics of fatigue crack initiation. International Journal of Fatigue, 57:58 - 72, 2013. Fatigue and Microstructure: A special issue on recent advances.

[25] Stephen D. Antolovich and Ronald W. Armstrong. Plastic strain localization in metals: origins and consequences. Progress in Materials Science, 59:1 - 160, 2014. 
[26] Beth E. Carroll, Todd A. Palmer, and Allison M. Beese. Anisotropic tensile behavior of ti-6al-4v components fabricated with directed energy deposition additive manufacturing. Acta Materialia, 87:309 - 320, 2015.

[27] Zhuqing Wang, Todd A. Palmer, and Allison M. Beese. Effect of processing parameters on microstructure and tensile properties of austenitic stainless steel $304 \mathrm{l}$ made by directed energy deposition additive manufacturing. Acta Materialia, 110:226 - 235, 2016.

[28] Alberto W. Mello, Andrea Nicolas, and Michael D. Sangid. Fatigue strain mapping via digital image correlation for ni-based superalloys: The role of thermal activation on cube slip. Materials Science and Engineering: A, 695:332 - 341, 2017.

[29] David Foehring, Huck Beng Chew, and John Lambros. Characterizing the tensile behavior of additively manufactured ti-6al-4v using multiscale digital image correlation. Materials Science and Engineering: A, 724:536 $-546,2018$.

[30] Michael D. Sangid, Todd A. Book, Diwakar Naragani, John Rotella, Priya Ravi, Alexander Finch, Peter Kenesei, Jun-Sang Park, Hemant Sharma, Jonathan Almer, and Xianghui Xiao. Role of heat treatment and build orientation in the microstructure sensitive deformation characteristics of in718 produced via slm additive manufacturing. Additive Manufacturing, 22:479 - 496, 2018.

[31] www.hoganas.com.

[32] www.beam-machines.com.

[33] U. de Oliveira, V. Ocelík, and J. Th M De Hosson. Analysis of coaxial laser cladding processing conditions. Surface and Coatings Technology, 197(2-3):127-136, 2005.

[34] www.flowwaterjet.com.

[35] http://www.gatan.com/products/sem-specimen-preparation/pecs-iisystem.

[36] Bing Pan, Kemao Qian, Huimin Xie, and Anand Asundi. Twodimensional digital image correlation for in-plane displacement and 
strain measurement: a review. Measurement Science and Technology, 20(6):062001, apr 2009.

[37] F. Hild and S. Roux. Digital image correlation: from displacement measurement to identification of elastic properties - a review. Strain, 42(2):69-80, 2006.

[38] L. Allais, M. Bornert, T. Bretheau, and D. Caldemaison. Experimental characterization of the local strain field in a heterogeneous elastoplastic material. Acta Metallurgica et Materialia, 42(11):3865 - 3880, 1994.

[39] M. Bornert, F. Brémand, P. Doumalin, J.-C. Dupré, M. Fazzini, M. Grédiac, F. Hild, S. Mistou, J. Molimard, J.-J. Orteu, L. Robert, Y. Surrel, P. Vacher, and B. Wattrisse. Assessment of digital image correlation measurement errors: Methodology and results. Experimental Mechanics, 49(3):353-370, Jun 2009.

[40] Pascal Doumalin. Microextensometrie locale par correlation d'images numeriques application aux etudes micromecaniques par microscopie electronique a balayage. $P h D, 2000$.

[41] H L Wei, J Mazumder, and T DebRoy. Evolution of solidification texture during additive manufacturing. Sci Rep, 5:16446, 2015.

[42] G. P. Dinda, A. K. Dasgupta, and J. Mazumder. Laser aided direct metal deposition of Inconel 625 superalloy: Microstructural evolution and thermal stability. Materials Science and Engineering A, 509(12):98-104, 2009.

[43] Lakshmi L. Parimi, G.A. Ravi, Daniel Clark, and Moataz M. Attallah. Microstructural and texture development in direct laser fabricated IN718. Materials Characterization, 89:102-111, 2014.

[44] Eric W Weisstein. Probability density function, http://mathworld.wolfram.com/probabilitydensityfunction.html.

[45] Michał Ziłetala, Tomasz Durejko, Marek Polanski, Izabela Kunce, Tomasz Płociński, Witold Zieliński, Magdalena Lazińska, Wojciech Stłepniowski, Tomasz Czujko, K.J. Kurzydłowski, and Zbigniew Bojar. The microstructure, mechanical properties and corrosion resistance of 
3161 stainless steel fabricated using laser engineered net shaping. Materials Science and Engineering: A, 677:1-10, 112016.

[46] Atasi Ghosh and N.P. Gurao. Effect of crystallographic texture on the planar anisotropy of ratcheting response in 316 stainless steel sheet. Materials and Design, 109:186 - 196, 2016.

[47] J. Yu, M. Rombouts, and G. Maes. Cracking behavior and mechanical properties of austenitic stainless steel parts produced by laser metal deposition. Materials and Design, 45:228 - 235, 2013.

[48] M.L. Griffith, D.M. Keicher, and J.T. Atwood. Free form fabrication of metallic components using laser engineering net shaping (lens). Proceedings of the Solid Freeform Fabrication Symposium, pages 125-132, 1996. cited By 1.

[49] T. DebRoy, H.L. Wei, J.S. Zuback, T. Mukherjee, J.W. Elmer, J.O. Milewski, A.M. Beese, A. Wilson-Heid, A. De, and W. Zhang. Additive manufacturing of metallic components - process, structure and properties. Progress in Materials Science, 92:112 - 224, 2018.

[50] Jorge Jorge. Influence of chemical composition on the mechanical properties of high strength steel weld metals for application in mooring components. International Journal of Engineering and Technical Research, 4:71-76, 022016.

[51] J.E. MacDonald, R.H.U. Khan, M. Aristizabal, K.E.A. Essa, M.J. Lunt, and M.M. Attallah. Influence of powder characteristics on the microstructure and mechanical properties of hipped cm247lc ni superalloy. Materials \& Design, 174:107796, 2019.

[52] Y.B. Zhang, A. Elbrønd, and F.X. Lin. A method to correct coordinate distortion in ebsd maps. Materials Characterization, 96:158 - 165, 2014.

[53] Fitzgerald Archibald Copyright (c) 2009. 